1 Transplantation of high fat fed mouse microbiota into zebrafish larvae identifies MyD88-

2 dependent acceleration of hyperlipidaemia by Gram positive cell wall components

4 Pradeep Manuneedhi Cholan ${ }^{1}$, Simone Morris ${ }^{1}$, Kaiming Luo $^{1}$, Jinbiao Chen ${ }^{2}$, Jade A Boland ${ }^{2}$, Geoff

5 W McCaughan ${ }^{2,3}$, Warwick J Britton ${ }^{1,4}$, Stefan H Oehlers ${ }^{1,5}$ *

61 Tuberculosis Research Program at the Centenary Institute, The University of Sydney,

7 Camperdown, NSW 2050, Australia

82 Liver Injury and Cancer Program at the Centenary Institute, The University of Sydney,

9 Camperdown, NSW 2050, Australia

${ }^{3}$ AW Morrow Gastroenterology and Liver Centre, Royal Prince Alfred Hospital, Camperdown, NSW

112050, Australia

$12{ }^{4}$ Department of Clinical Immunology, Royal Prince Alfred Hospital, Camperdown, NSW 2050,

13 Australia

$14{ }^{5}$ The University of Sydney, Discipline of Infectious Diseases \& Immunology and Marie Bashir 15 Institute, Camperdown, NSW 2050, Australia

Corresponding author: Dr Stefan Oehlers s.oehlers@centenary.org.au

ORCID: 0000-0003-0260-672X Twitter: @oehlerslab

21 Keywords: zebrafish, hyperlipidaemia, microbiota, pathobiont, myd88

\title{
23 Abstract
}

24 Gut dysbiosis is an important modifier of pathologies including cardiovascular disease but our

25 understanding of the role of individual microbes is limited. Here, we have used transplantation of 
mouse microbiota into microbiota-deficient zebrafish larvae to study the interaction between

members of a mammalian high fat diet-associated gut microbiota with a lipid rich diet challenge in

a tractable model species. We find zebrafish larvae are more susceptible to hyperlipidaemia when

exposed to the mouse high fat-diet-associated microbiota and that this effect can be driven by

two individual bacterial species fractionated from the mouse high fat-diet-associated microbiota.

We find Stenotrophomonas maltophilia increases the hyperlipidaemic potential of chicken egg

yolk to zebrafish larvae independent of direct interaction between S. maltophilia and the zebrafish

host. Colonisation by live, or exposure to heat-killed, Enterococcus faecalis accelerates

hyperlipidaemia via host Myd88 signalling. The hyperlipidaemic effect is replicated by exposure to

the Gram positive Toll-like receptor agonists peptidoglycan and lipoteichoic acid in a MyD88-

dependent manner. In this work, we demonstrate the applicability of zebrafish as a tractable host

for the identification of gut microbes that can induce conditional host phenotypes via microbiota

transplantation and subsequent challenge with a high fat diet.

\section{Introduction}

The metagenome encoded by the gut microbiome is an essential component of the animal

digestive system [1, 2]. Microbes can affect digestion directly through the breakdown of indigestible material such as fibre to short chain fatty acids and indirectly by stimulating the differentiation of the intestinal epithelium.

The microbiome is shaped by host and environment selective pressures [3, 4]. Lipid-rich Western diets that cause obesity in susceptible individuals are associated with gut dysbiosis which can drive an unwanted increase in nutrient absorption and epithelial leakiness [5]. Transplantation of human lean and obese microbiota into germ-free mice transmits these phenotypes across host 
51 microbe interactions through gnotobiotic and transplantation studies, there is a need for faster

52 and more accessible models that serve as functional screening tools for disease-associated

53 microbiota.

54

55 Zebrafish embryos require microbial colonisation during development for full physiological 56 digestive function and are a simple platform for studying host-microbiota-environment 57 interactions [7, 8]. Zebrafish embryos develop ex utero within a chorion that can be surface 58 sterilised and are tolerant of antibiotics in their media making them a technically simple model for 59 raising germ-free experimental subjects. Probiotic transfer has been shown to increase resistance 60 to pathogen colonisation [9]. The zebrafish gut microbiome is sensitive to challenge with a HFD,

61 feeding larval zebrafish a diet supplemented with $10 \%$ fat for 25 days altered the distribution of 62 bacterial phyla in the gut which correlated with increased expression of host inflammatory genes 63 compared to larvae fed the control diet [3].

64 Microbiota transplantations have been previously performed from mice and humans to zebrafish demonstrating the feasibility of using the zebrafish as an in vivo screening tool for studying the effects of defined microbiota on host physiology [10]. Here we use this technically simple model to rapidly identify specific members of the mouse HFD-associated microbiota that accelerate a dietinduced hyperlipidaemic phenotype in zebrafish embryos and the mechanisms by which these pathobionts interact with the zebrafish host.

\section{Results}

73 Transplantation of microbiota from HFD fed mice accelerates hyperlipidaemia in zebrafish 
75 Microbiome-depleted (MD) zebrafish embryos were exposed to mouse faecal microbiota

preparations generated from mice fed a conventional chow diet or HFD from 3-5 dpf (days post fertilization) and challenged with chicken egg yolk feeding from 5-7 dpf (Figure 1A).

We first investigated if colonisation with the control or HFD-fed mouse microbiota affected zebrafish intestinal physiology at $5 \mathrm{dpf}$ before the onset of exogenous feeding. The absorptive activity of zebrafish midgut lysosome-rich intestinal epithelial cells can be visualised by neutral red staining, previous studies have demonstrated that this phenotype requires microbial colonisation and is impeded by inflammation $[11,12]$. We used neutral red staining visually to examine the absorptive function of colonised embryos and observed higher staining in $5 \mathrm{dpf}$ larvae colonised with the HFD-fed microbiota compared to chow diet colonised controls (Figure 1B and 1C).

We next collected colonised zebrafish larvae and stained with Oil Red $O$ to visualise neutral lipids but did not observe any differences in lipid staining. To determine if colonisation with the HFD-fed mouse microbiota affected the zebrafish response to challenge with a chicken egg yolk challenge [13], we challenged $5 \mathrm{dpf}$ zebrafish with chicken egg yolk and stained with Oil Red $\mathrm{O}$ to visualise neutral lipids (Figure 1D). Zebrafish larvae colonised with the faecal microbiota from HFD fed mice had more vascular Oil Red $\mathrm{O}$ staining after 2 day of chicken egg yolk feeding challenge compared to zebrafish larvae colonised with the faecal microbiota from chow diet fed mice (Figure 1E).

These data demonstrate the responsiveness of MD zebrafish larvae to the transferable effects of dysbiotic mammalian gut microbiome-associated microbiota when challenged with a complex environmental stimulus in the form of lipid-rich feeding.

\footnotetext{
Identification of individual microbes with pathobiont activity
} 
100 To isolate individual species that would be amenable to in vitro handling and growth, faecal

101 homogenate supernatants were plated on LB agar and grown at $28^{\circ} \mathrm{C}$ to select for species that

102 would be easily handled and most likely to colonise zebrafish embryos (Figure $2 \mathrm{~A}$ ). The best

103 growing isolate in LB broth culture from two chow diet and HFD fed faecal preparations were

104 selected and sequenced. We identified Enterococcus faecalis strain YN771 (E.f on figures) and

105 Stenotrophomonas maltophilia strain CD103 (S.m) from HFD-fed mouse faecal lysate, and

106 Escherichia species PYCC8248 (E.s) and Escherichia coli strain Y15-3 (E.C) from chow diet-fed

107 mouse faecal lysate.

108

109

We colonised MD zebrafish larvae with individual isolates to determine if monoassociation could

110 replicate the effects of bulk microbiota transfer. To determine if exposure of larvae to

111 preparations of E. faecalis, S. maltophilia, or either of the E. coli strains resulted in gut colonisation

112 we dissected guts from rinsed $5 \mathrm{dpf}$ larvae after 2 days of monoassociation exposure and

113 recovered bacteria onto LB agar. All tested strains yielded recoverable colonisation levels of

114 approximately $100 \mathrm{CFU}$ per larval gut. Gnotobiotic zebrafish larvae colonised with E. faecalis or S.

115 maltophilia had increased Oil Red O staining compared to larvae colonised with either of the

116 Escherichia strains after chicken egg yolk challenge (Figure 2B).

118 Interestingly, colonisation with E. faecalis or S. maltophilia did not increase the absorptive activity

119 of the midgut intestinal epithelium compared to larvae colonised with either of the Escherichia

120 strains suggesting the absorptive phenotype seen in bulk microbiota transplant is the product of

121 multiple microorganisms or metabolites present in the complex mouse faecal microbiota 122 preparations (Figure $2 \mathrm{C}$ ). 
124 We next sought to confirm our observations using a second strain of each bacterial species, $E$.

125 faecalis UNSW 054400 type strain (E.f UNSW) and S. maltophilia yy01 (S.m yy01) isolated from

126 another mouse in the same facility. Colonisation of MD zebrafish embryos with either strain

127 replicated the hyperlipidaemic phenotype seen with our original isolates (Figure 2D). Interestingly,

128 the type UNSW 054400 strain increased hyperlipidaemia beyond that seen with our YN771 isolate

129 strain, while conversely the S. maltophilia yy01 strain was not as potent as our original CD103

130 strain, demonstrating strain-specific variability in our zebrafish embryo system.

132 Stenotrophomonas maltophilia can accelerate hyperlipidaemia by digesting food external to the

$133 \underline{\text { host }}$

134 To identify the mechanisms by which E. faecalis and S. maltophilia were accelerating HFD-induced

135 hyperlipidaemia in zebrafish larvae, we sought to investigate if the bacteria needed to be alive 136 and/or in contact with the host.

We adapted our gnotobiotic methodology to expose MD embryos to heat killed bacterial 139 preparations prior to chicken egg yolk feeding (Figure 3A). Exposure to heat-killed E. faecalis 140 replicated the live $E$. faecalis hyperlipidaemic phenotype when compared MD embryos that had 141 been exposed to either heat killed S. maltophilia or either of the Escherichia strains (Figure 3B). 142 Conversely, heat-killed S. maltophilia did not induce hyperlipidaemia in concert with chicken egg 143 yolk challenge.

145 We hypothesised S. maltophilia might interact with the chicken egg yolk independently of the host 146 colonisation. To test this hypothesis, we incubated chicken egg yolk with each of our 4 bacterial 147 isolates in conditions representative of the zebrafish embryo media and then sterilised the "pre148 digested" chicken egg yolk by autoclaving prior to feeding to MD zebrafish embryos (Figure 3C). 
149 Compared to either untreated chicken egg yolk, autoclaved chicken egg yolk, or chicken egg yolk

150 incubated with the other three bacterial isolates, the chicken egg yolk that had been incubated

151 with S. maltophilia increased hyperlipidaemia in zebrafish embryos (Figure 3D). A weaker, but

152 statistically significant, effect was also seen with chicken egg yolk that had been incubated with

153 Escherichia coli strain Y15-3 (E.c).

154

155 We next repeated this experiment in conventionally raised embryos and found the increased

156 hyperlipidaemic potential of S. maltophilia "pre-digested" chicken egg yolk was replicated in

157 larvae with a conventional microbiome (Figure 3E).

158

159 To examine the substrate specificity of S. maltophilia, we incubated commercially available fish 160 embryo food with Escherichia coli strain Y15-3 or S. maltophilia and observed an increase in body 161 size of only in embryos fed the commercial feed that had been "pre-digested" with S. maltophilia 162 compared to untreated commercial feed (Figure 3F).

164 Visual inspection of S. maltophilia "pre-digested" chicken egg yolk suspensions suggested S. 165 maltophilia had broken apart the chicken egg yolk resulting in smaller particles that could be more 166 easily ingested and altered the biochemical properties of the chicken egg yolk as the solution 167 contained much finer particles than for other treatments (Figure 3G). An intermediate phenotype 168 was seen in chicken egg yolk that had been "pre-digested" by E. coli strain Y15-3. CFU recovery 169 assays demonstrated higher growth of S. maltophilia than E. coli strain Y15-3 suggesting the better 170 growth of S. maltophilia may convert chicken egg yolk into components that could be digested by 171 zebrafish embryos (Figure $3 \mathrm{H}$ ). We performed nutritional panel and free fatty acid analyses of 172 chicken egg yolk that had been "pre-digested" by S. maltophilia or E. coli strain Y15-3 as an 173 additional control (Table 1). These analyses revealed only a modest increase in energy content by 
$174 E$ coli strain $\mathrm{Y} 15-3$ and S. maltophilia, and an increase in total fat content made up of

175 monounsaturated and saturated fatty acids in S. maltophilia-incubated samples that was not seen

176 in control or E. coli strain Y15-3-incubated samples.

177

178 These data illustrate a colonisation-independent mechanism by which S. maltophilia may enhance

179 lipid uptake in zebrafish larvae by modifying food in the aqueous environment external to the 180 host.

181

182 Enterococcus faecalis accelerates hyperlipidaemia via host MyD88-mediated signalling

183 Our analyses had shown E. faecalis did not need to be alive but did need to be pre-associated with

184 the hatching zebrafish larvae to accelerate hyperlipidaemia suggesting colonisation of the gut or

185 other mucosal surfaces and subsequent recognition by the host may been necessary to accelerate

186 hyperlipidaemia. Host innate immune signalling via the Myd88 adaptor protein is essential for the

187 zebrafish intestinal epithelium to respond to microbial colonisation [14].

189 We performed knockdown of host myd88 expression using multiple CRISPR-Cas9 gRNAs (Figure

190 4A). Host myd88 expression was necessary for transducing the E. faecalis-induced hyperlipidaemic

191 signal as myd88 crispants had significantly less vascular Oil Red O staining than scrambled 192 gRNA/Cas9-injected control embryos after colonisation with E. faecalis and chicken egg yolk 193 challenge (Figure 4B).

195 Gram positive cell wall components accelerate hyperlipidaemia in zebrafish embryos

196 E. faecalis is a Gram-positive bacterium. To determine if E. faecalis-driven hyperlipidaemia was 197 due to a conserved Gram-positive cell wall component, we initially compared the hyperlipidaemic 198 potential of heat-killed E. faecalis to heat killed Staphylococcus xylosus (S.x), another Gram- 
199

200

201

202

203

204

205

206

207

208

209

210

211

212

213

214

215

216

217

218

219

220

221

222

positive bacterium obtained from the faecal microbiota of a mouse from the same facility. We found exposure of zebrafish larvae to heat-killed S. xylosus replicated the hyperlipidaemic effect of heat-killed E. faecalis after chicken egg yolk challenge (Figure 5A).

Next we directly soaked MD larvae in a sublethal dose of $25 \mu \mathrm{g} / \mathrm{mL}$ purified lipoteichoic acid (LTA) or peptidoglycan (PGL), which are major components of the Gram-positive cell wall, prior to challenge with chicken egg yolk feeding [15]. Either one of these purified ligands were able to accelerate hyperlipidaemia in MD larvae (Figure 5B).

To test the requirement for intact $E$. faecalis PGL to accelerate hyperlipidaemia, we exposed MD larvae to lysozyme-digested heat-killed E. faecalis prior to chicken egg yolk feeding. Lysozyme digestion ablated the ability of heat-killed E. faecalis to accelerate hyperlipidaemia (Figure 5C).

To determine if host innate immune signalling via the Myd88 adaptor protein transduced the LTA or PGL-induced signal that accelerates hyperlipidaemia, we again knocked down myd88 using a pooled CRISPR-Cas9 gRNA approach and exposed crispants to LTA or PGL. Depletion of host myd88 ablated the sensitivity of embryos to LTA and PGL-induced accelerated hyperlipidaemia (Figure 5D).

Together, these experiments demonstrate larval zebrafish lipid metabolism is sensitive to the presence of Gram-positive bacterial cell wall components via Myd88-mediated host signalling pathways.

\section{Discussion}


223 Our study demonstrates the utility of the gnotobiotic zebrafish platform to screen donor

224 microbiota samples for transplantable biological activities in combination with an exogenous

225 environmental factor. The addition of an exogenous trigger to the experimental system is an

226 important permutation that allows the identification of microbes whose interactions with the host

227 only become apparent in combination with an environmental challenge such as diet.

We applied the gnotobiotic zebrafish platform to explore the diet-hyperlipidaemia axis as there is

a growing interest in microbiome studies within the cardiovascular disease field [16]. The

231 gnotobiotic zebrafish platform represents a disruptive technology that could be adapted to

232 identify pathobiont species from human cardiovascular disease patients and as a "first pass"

233 platform in mechanistic studies with appropriate zebrafish models of cardiovascular pathology.

234

235 Our investigation of E. faecalis-accelerated hyperlipidaemia uncovered a surprising role of Gram-

236 positive cell wall component-triggered Myd88 signalling in zebrafish embryo lipid metabolism. The

237 presence of this response in zebrafish embryos has broad implications for the use of zebrafish

238 embryos to study transplanted mammalian microbiota as it may not be representative of the

239 mammalian response to colonisation with Gram positive organisms. Most zebrafish embryo mono-

240 association studies have been carried out with Gram negative organisms including E. coli, $A$.

241 veronii, V. cholerae, and P. aeruginosa [17-19].

243 The comparison of heat killed E. faecalis to heat killed S. xylosus demonstrated an increased ability

244 of heat killed E. faecalis to accelerate diet-induced hyperlipidaemia. This suggests that there is 245 variability in hyperlipidaemia accelerating potential amongst Gram positive organisms in zebrafish 246 embryos. The basis of this difference could be further explored using model Gram positive 247 organisms and Gram-positive organisms that are natural commensals in the zebrafish gut. A recent 
248

249

250

251

252

253

254

255

256

257

258

259

260

261

262

263

264

265

266

267

268

269

270

271

272

paper by Griffin et al. has demonstrated a role for E. faecalis SagA enzyme in producing immunostimulatory muropeptides [20], as we have previously demonstrated a conserved function of the zebrafish NOD orthologs it is possible that SagA-mediated production of muropeptides from bacterial peptidoglycan contributes to our hyperlipidaemia-accelerating phenotype.

Caenorhabditis elegans fed with S. maltophilia accumulate neutral lipids within intracellular lipid droplets driven by a bacterially-encoded mechanism that is independent of the innate immune response [21]. Our finding that two distinct mouse-associated S. maltophilia strains were able to increase the hyperlipidaemic potential of chicken egg yolk suggests the digestive ability of $S$. maltophilia is potentially conserved between strains and potentially between host species. Previous studies have identified S. maltophilia strains within zebrafish gut microbiomes [9], these zebrafish-associated strains could be analysed to determine if increased lipid uptake is a consequence of natural host-S. maltophilia pairs and for comparative studies to identify the food modifying mechanisms employed by our S. maltophilia CD103 strain isolate.

We also found an embryo growth-enhancing effect of pre-digesting commercially available zebrafish embryo feed with S. maltophilia which suggests S. maltophilia could have commercial applications as an aquaculture feed additive. The use of S. maltophilia as a feed additive is potentially risky as this organism is associated with opportunistic infections in humans and is resistant to a wide range of antibiotics so care should be taken to avoid cross over into the food chain $[22,23]$.

As a proof-of-principle study, our work demonstrates the feasibility of studying the interaction of bacterial species transplanted from a mammalian host with an environmental factor to result in a conditional phenotype in the tractable zebrafish model system. Further work is required to 
273 examine effects of the two pathobiont species identified in our study on mammalian models of

274 hyperlipidaemia and to correlate their colonisation with the progression of cardiovascular disease

275 phenotypes in mammalian models and human samples.

276

277 Methods

278 Zebrafish handling

279 Adult zebrafish were housed at the Centenary Institute (Sydney Local Health District Animal

280 Welfare Committee Approval 17-036). Zebrafish embryos were obtained by natural spawning and

281 conventionally raised embryos were maintained in $\mathrm{E} 3$ media at $28^{\circ} \mathrm{C}$.

282

283 Generation of microbiome-depleted (MD) zebrafish embryos

284 Microbiome-depleted (MD) zebrafish were created and maintained as previously described [24].

285 Briefly, freshly laid embryos were rinsed with $0.003 \% \mathrm{v} / \mathrm{v}$ bleach in sterile E3 and rinsed 3 times

286 with sterile E3. Bleached embryos were raised in sterile E3 supplemented with $50 \mu \mathrm{g} / \mathrm{mL}$ ampicillin

287 (Sigma), $5 \mu \mathrm{g} / \mathrm{mL}$ kanamycin (Sigma) and $250 \mathrm{ng} / \mathrm{mL}$ amphotericin B (Sigma) in sterile tissue

288 culture flasks. Dead embryos and chorions were aseptically removed at one and three $\mathrm{dpf}$ 289 respectively.

290

291 Conventionalised zebrafish were used as a control, where $3 \mathrm{dpf}$ MD zebrafish were inoculated 292 with system water from the aquarium at Centenary Institute.

293

294 Generation of mouse faecal microbiota specimens

295 Mice were housed at the Centenary Institute (Sydney Local Health District Animal Welfare

296 Committee Approval 2018/016). C57BL/6J mice were housed in a pathogen-free and temperature-

297 controlled environment, with 12 hours of light and 12 hours of darkness, and free access to food 
and water. Mice were provided with a High Fat Diet (HFD) or chow from 6 to 30 weeks of age. The

HFD was prepared in-house based on rodent diet no. D12451 (Research Diets New Brunswick,

USA) and its calories were supplied as: fat $45 \%$, protein $20 \%$, and carbohydrate $35 \%$ [25]. The

chow diet was commercially produced by Speciality Feeds as "Irradiated Rat and Mouse Diet" and

its calories were supplied as: fat $12 \%$, protein $23 \%$, and carbohydrate $65 \%$.

303

304 Faecal pellets were collected from mice that were housed in different cages. Individual faecal pellets were collected into sterile $1.7 \mathrm{~mL}$ microcentrifuge tubes and homogenised in $1 \mathrm{~mL}$ of sterile

306 E3 by pipetting. Homogenised specimens were centrifuged at $500 \mathrm{G}$ for 2 minutes to sediment

307 fibrous material and the supernatant was collected. Supernatants were supplemented with

308 glycerol to a final concentration of $25 \% \mathrm{v} / \mathrm{v}$, aliquoted, and frozen at $-80^{\circ} \mathrm{C}$ for experimental use.

310 Colonisation of zebrafish with mouse faecal microbiota

311 MD zebrafish were colonised at $3 \mathrm{dpf}$ by transfer into sterile E3 and addition of $200 \mu \mathrm{L}$ thawed

312 faecal homogenate supernatant. At $5 \mathrm{dpf}$, embryos were rinsed with E3 and placed on a chicken 313 egg yolk diet.

315 Neutral red staining and morphology measurements

316 Neutral red staining was performed as previously described [26]. Briefly, 2.5? $\mu \mathrm{g} / \mathrm{mL}$ neutral red 317 was added to the media of $4 \mathrm{dpf}$ embryos and incubated overnight. Embryos were rinsed with 318 fresh E3 to remove unbound neutral red and live imaged on a Leica M205FA microscope with a 319 consistent zoom between specimens in a single experiment. The area of neutral red-stained 320 midgut was measured in pixels using ImageJ. Body size was measured in pixels using ImageJ. 
323 High fat diet challenge with chicken egg yolk was performed as previously described [13]. Briefly, 5

$324 \mathrm{dpf}$ zebrafish embryos were placed in an E3 solution containing $0.05 \% \mathrm{w} / \mathrm{v}$ emulsified hard boiled

325 chicken egg yolk in glass beakers. Beakers were housed in a $28^{\circ} \mathrm{C}$ incubator with a $14: 10$ hour

326 light:dark cycle. The emulsified hard boiled chicken egg yolk solution was changed daily.

Oil Red O staining assay

Oil Red O staining and analysis was performed as previously described $[13,27,28]$. Briefly, $7 \mathrm{dpf}$

embryos were fixed overnight at $4^{\circ} \mathrm{C}$ in $4 \%$ paraformaldehyde, rinsed with PBS, and rinsed stepwise through to propylene glycol. Embryos were stained with filtered $0.5 \%(\mathrm{w} / \mathrm{v})$ Oil Red 0 dissolved in propylene glycol overnight at room temperature. Unbound dye was removed by washing with propylene glycol and embryos were rinsed stepwise through to PBS for imaging.

Embryos were imaged on a Leica M205FA microscope. Experimental batches of colour images were analysed in ImageJ by adjusting the colour threshold function to eliminate non-red signal,

this output was then converted to a binary mask and the tail region posterior to the swim bladder was selected to measure the area of particles.

340 Isolation, identification, and handling of bacterial isolates from mouse faecal microbiota samples

341 Faecal homogenate supernatants were plated onto LB Agar (Amyl Media) and incubated at $28^{\circ} \mathrm{C}$ 342 for two days. Individual isolates were grown in broth culture in Luria Broth (Miller's LB Broth Base, 343 Thermofisher) at $28^{\circ} \mathrm{C}$ overnight with 200 RPM shaking.

345 For identification, bacteria were harvested from broth culture and subjected to PCR using 346 universal 16S primers (50-3ㄹ) Fw Rv. PCR products were sequenced by Sanger Sequencing (AGRF) 
347 and NCBI records were searched by BLAST to identify the closest matching bacterial strain by

348 sequence identity.

350 For gnotobiotic experiments, bacteria were harvested from overnight broth cultures and

351 resuspended in sterile E3 zebrafish embryo media at a concentration of OD600 0.2. MD zebrafish

352 in autoclaved E3 were then inoculated with the bacterial suspension at a ratio of 1:200.

353

354 For heat killed bacterial inoculations, bacteria were resuspended in E3 at a concentration of 355 OD600 0.2 and heat-killed in a $95^{\circ} \mathrm{C}$ heat block for 30 minutes. Heat killed bacterial solutions were 356 then added at 1:200 ratio to $3 \mathrm{dpf}$ MD zebrafish.

357

358 For purified ligand exposure, $3 \mathrm{dpf}$ MD zebrafish were soaked in $25 \mu \mathrm{g} / \mathrm{mL}$ lipoteichoic acid 359 (Sigma) or $25 \mu \mathrm{g} / \mathrm{mL}$ peptidoglycan (Sigma).

360

361 For lysozyme digestion of heat-killed E. faecalis, a suspension of heat-killed bacteria was incubated 362 with $50 \mu \mathrm{g} / \mathrm{mL}$ lysozyme (Sigma) at $37^{\circ} \mathrm{C}$ for $6 \mathrm{~h}$ before being used to inoculate $3 \mathrm{dpf} \mathrm{MD}$ zebrafish 363 at a ratio of 1:200.

Digestion of chicken egg yolk

$36620 \mathrm{~g}$ hard-boiled chicken egg yolk was mixed with $40 \mathrm{~mL}$ E3 and emulsified using a Branson Digital 367 Sonifier sonicator. Emulsified egg yolk was inoculated with bacterial isolates in E3 (described 368 above) at a 1:200 ratio. Egg yolk mixture was then placed in a shaker at $28^{\circ} \mathrm{C}$ at $200 \mathrm{rpm}$ and for $36948 \mathrm{~h}$. Samples were then autoclaved prior to feeding experiments. 
372 "Pre-digested" samples were autoclaved and mailed to Australian Laboratory Services (VIC,

373 Australia) for commercial grade nutritional analyses.

374

375 Gene knockdown with CRISPR-Cas9

376 gRNA templates for myd88 $\quad\left(5^{`}-43^{\prime}\right): \quad$ Target 1

377 TAATACGACTCACTATAGgCAGTtTCCGAAAGAAACTGTTtTAGAGCTAGAAATAGC, Target 2

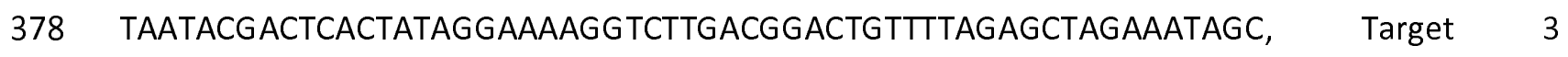

379 TAATACGACTCACTATAGGAACTGTTTGATCATCTCGGTTTAAGAGCTAGAAATAGC, $\quad$ Target 4

380 TAATACGACTCACTATAGGTTTTTTCGATAAGCTCACGTTTTAGAGCTAGAAATAGC. gRNA was

381 synthesized as previously described [29].

382

383 A 1:1 solution of gRNA and $500 \mu \mathrm{g} / \mathrm{mL}$ of Cas9 nuclease V3 (Integrated DNA Technology, Sigma, or

384 or Sydney Analytical) was prepared with phenol red dye (Sigma, P0290). Freshly laid eggs were

385 collected from breeding tanks and the solution was injected in the yolk sac of the egg before the 386 emergence of the first cell with a FemtoJet $4 i$ (Eppendorf).

387

388 Knockdown efficacy was monitored by RT-qPCR as previously described [30]. myd88-specific 389 primers (5’3'): Fw ACAGGGACTGACACCTGAGA, Rv GACGACAGGGATTAGCCGTT.

390

391 To derive MD crispant embryos, injected embryos were placed in E3 containing ampicillin, 392 kanamycin and amphotericin B as described previously. Bleaching injected embryos caused high 393 mortality rates. 
All statistical analyses (t-tests and ANOVA where appropriate) were performed using GraphPad

Prism 8. Outliers were removed using ROUT, with $Q=1 \%$. All data shown are representative of at least 2 biological replicates.

399

400

\section{Data availability}

401 Source data are provided with this paper.

402

Raw image and analysis data is archived for 10 years by The Centenary Institute and available on request from the corresponding author (Sydney, Australia).

404

\section{Acknowledgements}

406 Funding: Australian National Health and Medical Research Council Project Grant APP1099912; The

407 University of Sydney Fellowship G197581; NSW Ministry of Health under the NSW Health Early-

Mid Career Fellowships Scheme H18/31086 to SHO. Australian National Health and Medical

Research Council Centres of Research Excellence Grant APP1153493 to WJB. The funders had no

role in study design, data collection and analysis, decision to publish, or preparation of the manuscript.

The E. faecalis UNSW 054400 type strain was provided by Dr Laurence Marcia.

The authors acknowledge the facilities and the technical assistance of Dr Angela Kurz at the 
421

422

423

424

425

426

427

428

429

430

431

432

433

434

435

436

437

438

439

440

441

442

443

444

445

446

447

448

449

450

451

452

453

454

455

456

457

458

459

460

461

462

463

464

465

466

467

468

\section{References}

1. Sekirov, I., et al., Gut microbiota in health and disease. Physiol Rev, 2010. 90(3): p. 859-904.

2. Backhed, F., et al., Mechanisms underlying the resistance to diet-induced obesity in germfree mice. Proc Natl Acad Sci U S A, 2007. 104(3): p. 979-84.

3. Arias-Jayo, N., et al., High-Fat Diet Consumption Induces Microbiota Dysbiosis and Intestinal Inflammation in Zebrafish. Microb Ecol, 2018. 76(4): p. 1089-1101.

4. Rawls, J.F., et al., Reciprocal gut microbiota transplants from zebrafish and mice to germfree recipients reveal host habitat selection. Cell, 2006. 127(2): p. 423-33.

5. Murphy, E.A., K.T. Velazquez, and K.M. Herbert, Influence of high-fat diet on gut microbiota: a driving force for chronic disease risk. Curr Opin Clin Nutr Metab Care, 2015. 18(5): p. 515-20.

6. Ridaura, V.K., et al., Gut microbiota from twins discordant for obesity modulate metabolism in mice. Science, 2013. 341(6150): p. 1241214.

7. Lopez Nadal, A., et al., Feed, Microbiota, and Gut Immunity: Using the Zebrafish Model to Understand Fish Health. Front Immunol, 2020. 11: p. 114.

8. Brinkmann, B.W., et al., Colonizing microbiota protect zebrafish larvae against silver nanoparticle toxicity. Nanotoxicology, 2020. 14(6): p. 725-739.

9. Stressmann, F.A., et al., Mining zebrafish microbiota reveals key community-level resistance against fish pathogen infection. ISME J, 2021. 15(3): p. 702-719.

10. Valenzuela, M.J., et al., Evaluating the Capacity of Human Gut Microorganisms to Colonize the Zebrafish Larvae (Danio rerio). Front Microbiol, 2018. 9: p. 1032.

11. Bates, J.M., et al., Distinct signals from the microbiota promote different aspects of zebrafish gut differentiation. Dev Biol, 2006. 297(2): p. 374-86.

12. Oehlers, S.H., et al., A chemical enterocolitis model in zebrafish larvae that is dependent on microbiota and responsive to pharmacological agents. Dev Dyn, 2011. 240(1): p. 288-98.

13. Morris, S., et al., Glucose inhibits haemostasis and accelerates diet-induced hyperlipidaemia in zebrafish larvae. Sci Rep, 2021.

14. Cheesman, S.E., et al., Epithelial cell proliferation in the developing zebrafish intestine is regulated by the Wnt pathway and microbial signaling via Myd88. Proc Natl Acad Sci U S A, 2011. 108 Suppl 1: p. 4570-7.

15. Novoa, B., et al., LPS response and tolerance in the zebrafish (Danio rerio). Fish Shellfish Immunol, 2009. 26(2): p. 326-31.

16. Kaye, D.M., et al., Deficiency of Prebiotic Fiber and Insufficient Signaling Through Gut Metabolite-Sensing Receptors Leads to Cardiovascular Disease. Circulation, 2020. 141(17): p. 1393-1403.

17. Tan, F., et al., The Responses of Germ-Free Zebrafish (Danio rerio) to Varying Bacterial Concentrations, Colonization Time Points, and Exposure Duration. Front Microbiol, 2019. 10: p. 2156.

18. Weitekamp, C.A., et al., Monoassociation with bacterial isolates reveals the role of colonization, community complexity and abundance on locomotor behavior in larval zebrafish. Anim Microbiome, 2021. 3(1): p. 12.

19. Robinson, C.D., et al., Experimental bacterial adaptation to the zebrafish gut reveals a primary role for immigration. PLoS Biol, 2018. 16(12): p. e2006893.

20. Griffin, M.E., et al., Enterococcus peptidoglycan remodeling promotes checkpoint inhibitor cancer immunotherapy. Science, 2021. 373(6558): p. 1040-1046.

21. Xie, K., et al., Dietary $<e m>$ S. maltophilia $</$ em $>$ promotes fat storage by enhancing lipogenesis and ER-LD contacts in <em>C. elegans</em>. bioRxiv, 2020: $\mathrm{p}$. 2020.04.29.067793. 
469

470

471

472

473

474

475

476

477

478

479

480

481

482

483

484

485

486

487

488

489

490

491

492

493

494

495

496

497

498

499

500

501

22. Brooke, J.S., Stenotrophomonas maltophilia: an emerging global opportunistic pathogen. Clin Microbiol Rev, 2012. 25(1): p. 2-41.

23. Pathmanathan, A. and G.W. Waterer, Significance of positive Stenotrophomonas maltophilia culture in acute respiratory tract infection. Eur Respir J, 2005. 25(5): p. 911-4.

24. Melancon, E., et al., Best practices for germ-free derivation and gnotobiotic zebrafish husbandry. Methods Cell Biol, 2017. 138: p. 61-100.

25. Henderson, J.M., et al., Multiple liver insults synergize to accelerate experimental hepatocellular carcinoma. Sci Rep, 2018. 8(1): p. 10283.

26. Oehlers, S.H., et al., Chemically induced intestinal damage models in zebrafish larvae. Zebrafish, 2013. 10(2): p. 184-93.

27. Fang, L., et al., In vivo visualization and attenuation of oxidized lipid accumulation in hypercholesterolemic zebrafish. J Clin Invest, 2011.

28. Johansen, M.D., et al., Mycobacterium marinum infection drives foam cell differentiation in zebrafish infection models. Dev Comp Immunol, 2018. 88: p. 169-172.

29. Wu, R.S., et al., A Rapid Method for Directed Gene Knockout for Screening in GO Zebrafish. Dev Cell, 2018. 46(1): p. 112-125 e4.

30. Cholan, P.M., et al., Conserved anti-inflammatory effects and sensing of butyrate in zebrafish. Gut Microbes, 2020. 12(1): p. 1-11.

\section{Figure Legends}

Figure 1: Transplantation of microbiota from HFD-fed mice accelerates hyperlipidaemia in zebrafish embryos.

(a) Schematic describing method of faecal microbiome inoculation in MD zebrafish and chicken egg yolk challenge diet. (b) Representative images of neutral red staining in mid-gut of $5 \mathrm{dpf}$ zebrafish embryos. Red brackets indicate mid-gut region used for quantification. (c) Quantification of neutral red staining area in mid-gut of $5 \mathrm{dpf}$ zebrafish embryos. (d) Representative images of Oil Red $\mathrm{O}$ staining of $7 \mathrm{dpf}$ chow-fed and HFD-fed mouse faecal microbiome-inoculated zebrafish embryos. Red brackets indicate tail region posterior to the swim bladder used for quantification (e) Quantification of trunk vascular Oil Red O staining from the tail region posterior to the swim bladder in MD zebrafish inoculated with mouse faecal microbiota and challenged with chicken egg yolk diet from 5-7 dpf. Scale bar represents $500 \mu \mathrm{m}$. Results are expressed as mean \pm SD.

Figure 2: Identification of individual microbes with pathobiont activity. 
502 (a) Schematic describing workflow to isolate Escherichia species PYCC8248 (E.s), Escherichia coli

503 strain Y15 (E.C), and Enterococcus faecalis strain YN771 (E.f) and inoculate into MD zebrafish with

504 subsequent chicken egg yolk diet challenge. (b) Quantification of trunk vascular Oil Red O staining

505 from the tail region posterior to the swim bladder in MD zebrafish inoculated with bacterial

506 isolates E.S, E.C, and E.f and challenged with a chicken egg yolk diet from 5-6 dpf. (c) Area of

507 neutral red stained in the mid-gut of $5 \mathrm{dpf}$ gnotobiotic zebrafish embryos mono-associated with

508 bacterial isolates E.S, E.C, and E.f. (d) Quantification of trunk vascular Oil Red O staining from the

509 tail region posterior to the swim bladder in MD zebrafish inoculated with bacterial isolates $E$. $f$ and

510 E. faecalis UNSW 054400 type strain (E.f UNSW) then challenged with a chicken egg yolk diet from

$5115-6$ dpf. Results are expressed as mean \pm SD.

512

513 Figure 3: Stenotrophomonas maltophilia accelerates hyperlipidaemia by digesting food.

514 (a) Schematic describing creation of "pre-digested" chicken egg yolk by incubation with bacterial 515 isolates E.S, E.C, S.m, and E.f, autoclaving, for feeding to 5-7 dpf zebrafish embryos. (b)

516 Quantification of trunk vascular Oil Red O staining from the tail region posterior to the swim 517 bladder in MD zebrafish fed "pre-digested" egg yolk with bacterial isolates E.s, E.C, S.m, and E.f 518 from 5-7 dpf. (c) Quantification of trunk vascular Oil Red O staining from the tail region posterior 519 to the swim bladder in conventionally raised zebrafish fed "pre-digested" egg yolk with bacterial 520 isolate S.m from 5-7 dpf. (d) Quantification of trunk vascular Oil Red O staining from the tail region 521 posterior to the swim bladder in conventionally raised zebrafish fed "pre-digested" fish embryo 522 food with bacterial isolates E.C and S.m from 5-7 dpf. (e) Representative images of "pre-digested" 523 egg yolk with bacterial isolates E.C and S.m red brackets indicate fraction of the water column 524 containing large particulates after autoclaving. (f) CFU recovery from chicken egg yolk "pre525 digestion" reactions. Results are expressed as mean \pm SD. 
527 Figure 4: Enterococcus faecalis (E.f) accelerates hyperlipidaemia via host MyD88-mediated 528 signalling.

529 (a) Schematic describing inoculation of MD zebrafish embryos with heat-killed bacterial isolates 530 E.s, E.C, and E.f from 3-5 dpf, followed by chicken egg yolk diet challenge from 5-7 dpf. (b)

531 Quantification of trunk vascular Oil Red O staining from the tail region posterior to the swim 532 bladder in MD zebrafish inoculated with heat killed bacterial isolates E.S, E.C, and E.f and 533 challenged with a chicken egg yolk diet. (c) Quantification of myd88 expression in zebrafish 534 embryos injected with myd88-targeting CRISPR-Cas9 complexes at $5 \mathrm{dpf}$. Each dot represents a 535 biological replicate of at least 10 embryos. (d) Quantification of trunk vascular Oil Red O staining 536 from the tail region posterior to the swim bladder in control scrambled and myd88 crispant 537 embryos exposed to E.f and challenged with a chicken egg yolk diet. Results are expressed as 538 mean $\pm S D$.

540 Figure 5: Gram-positive cell wall components accelerate hyperlipidaemia in zebrafish embryos.

541 (a) Quantification of trunk vascular Oil Red O staining from the tail region posterior to the swim 542 bladder in MD zebrafish inoculated with heat killed bacterial isolates E.f and Staphylococcus 543 xylosus (S.x) from 3-5 dpf and challenged with a chicken egg yolk diet from 5-7 dpf. (b) 544 Quantification of trunk vascular Oil Red O staining from the tail region posterior to the swim 545 bladder in MD zebrafish pre-incubated with LTA or peptidoglycan from 3-5 dpf before co546 challenge with chicken egg yolk diet from 5-7 dpf. (c) Quantification of trunk vascular Oil Red O 547 staining from the tail region posterior to the swim bladder in MD zebrafish pre-incubated with 548 lysozyme-treated heat killed E.f from 3-5 dpf and challenged with a chicken egg yolk diet from 5-7 549 dpf. (d) Quantification of trunk vascular Oil Red O staining from the tail region posterior to the 550 swim bladder in control scrambled and myd88 crispant embryos pre-incubated with LTA or 
bioRxiv preprint doi: https://doi.org/10.1101/2021.04.28.441900; this version posted September 21, 2021. The copyright holder for this preprint (which was not certified by peer review) is the author/funder, who has granted bioRxiv a license to display the preprint in perpetuity. It is made available under aCC-BY-NC-ND 4.0 International license.

551 peptidoglycan from 3-5 dpf before co-challenge with chicken egg yolk diet from 5-7 dpf. Results

552 are expressed as mean \pm SD.

553 
Chow-fed mice homogenate LB agar @

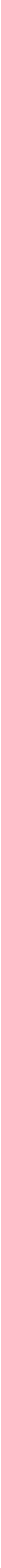




\section{Heat inactivated}

\section{MD zebrafish} isolate

\section{Egg yolk}

\section{inoculation}

$0 \mathrm{dpf}$

$3 \mathrm{dpf}$

$5 \mathrm{dpf}$

$7 \mathrm{dpf}$

B

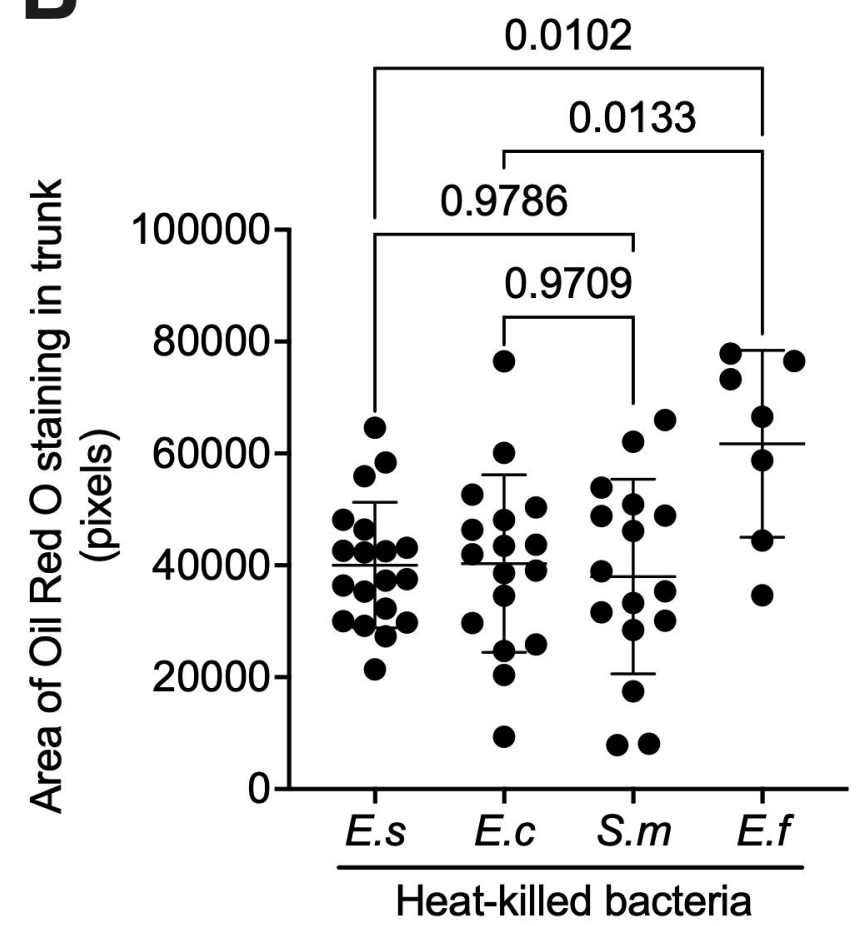

C

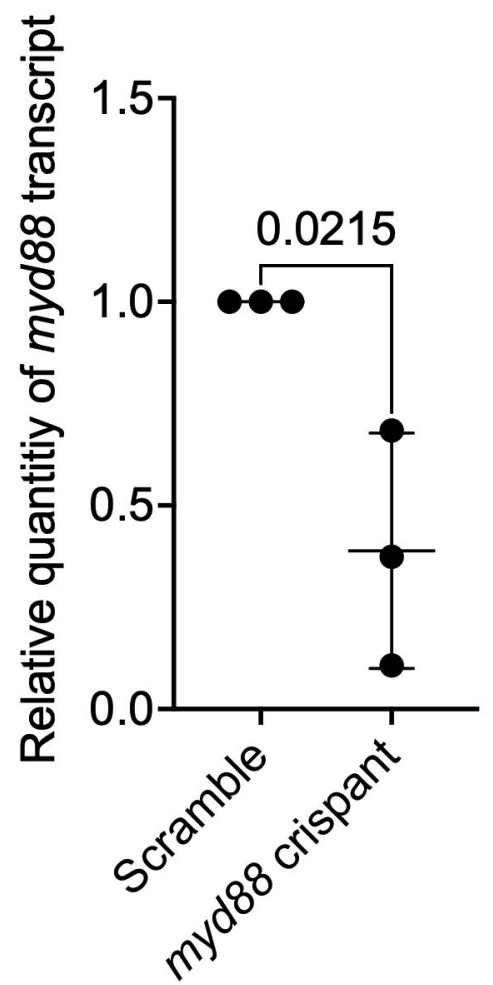

D

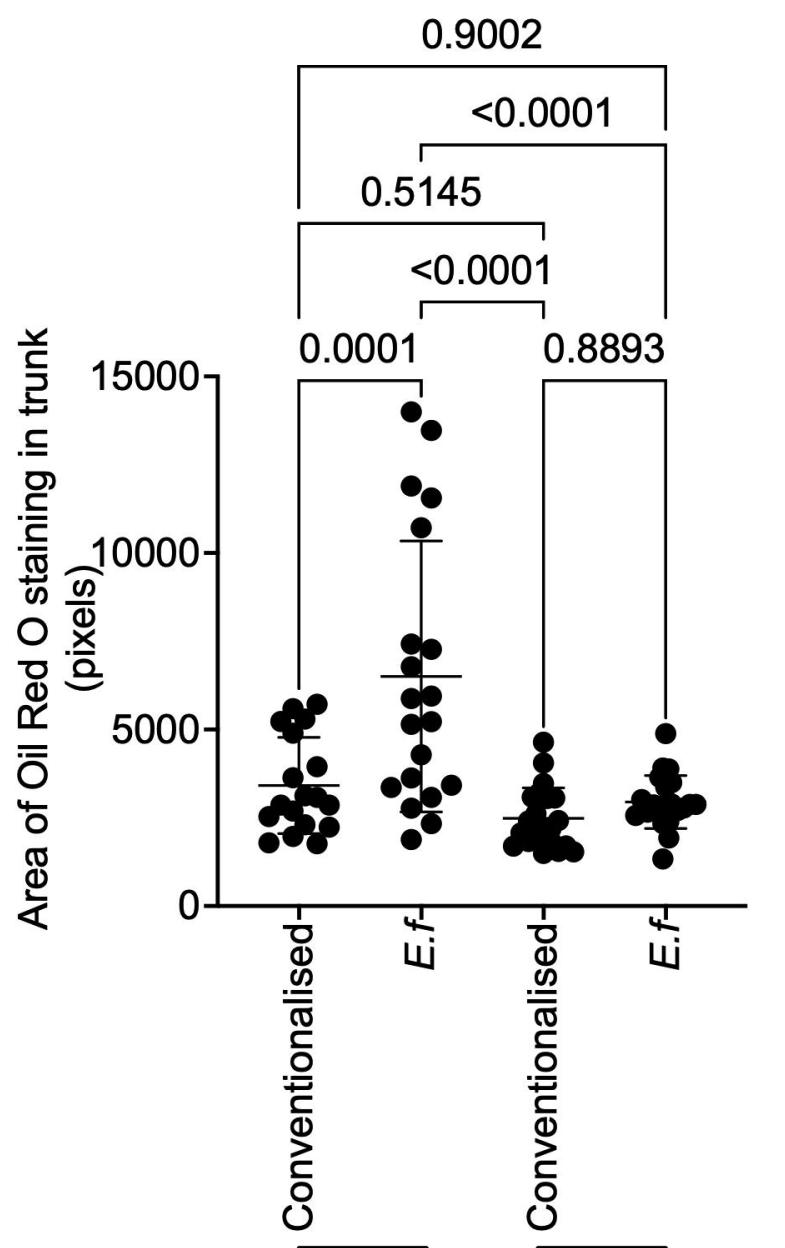

scrambled myd88 crispant 
A

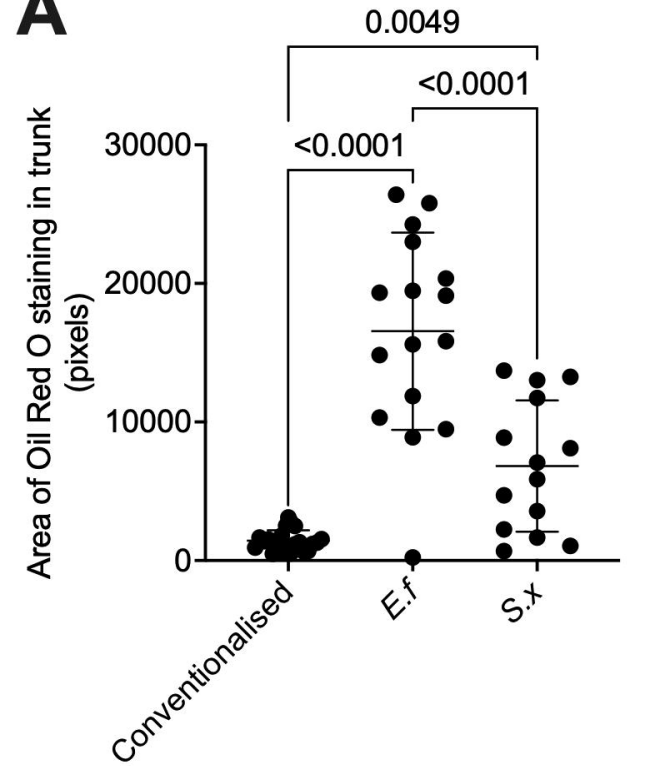

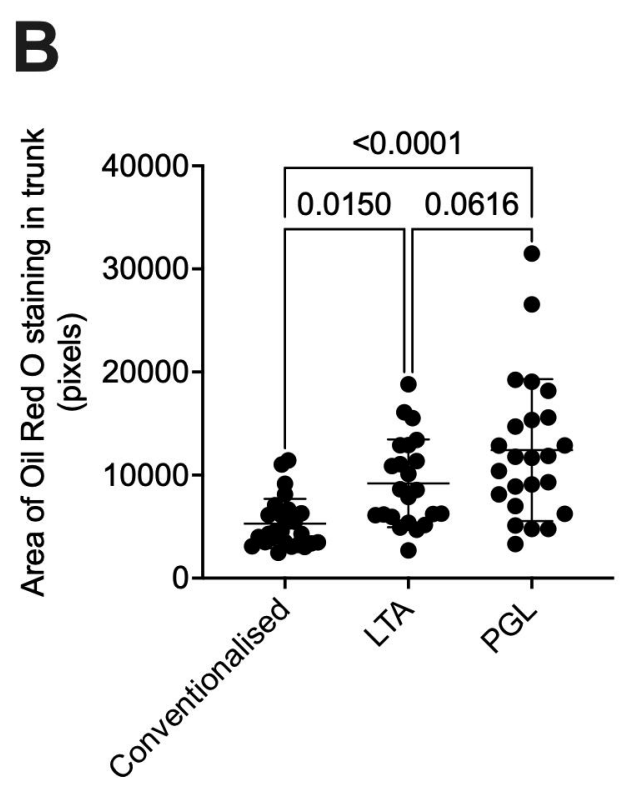

C

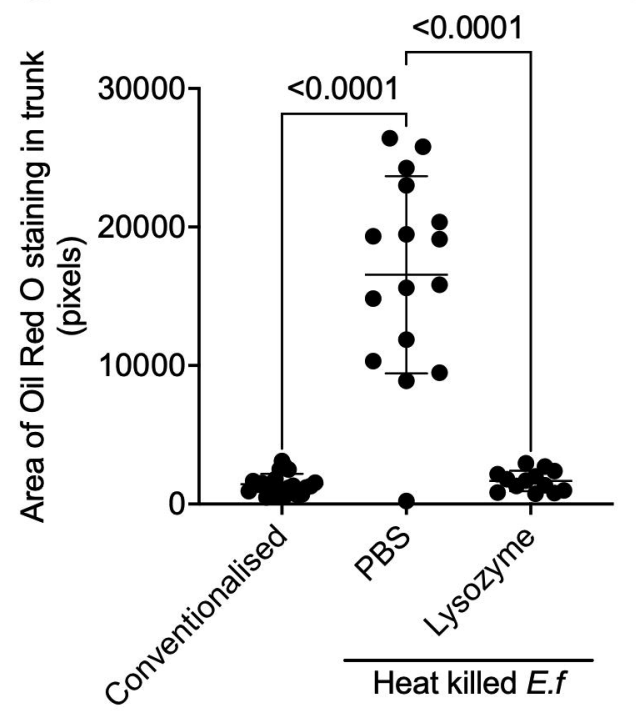

0.0003

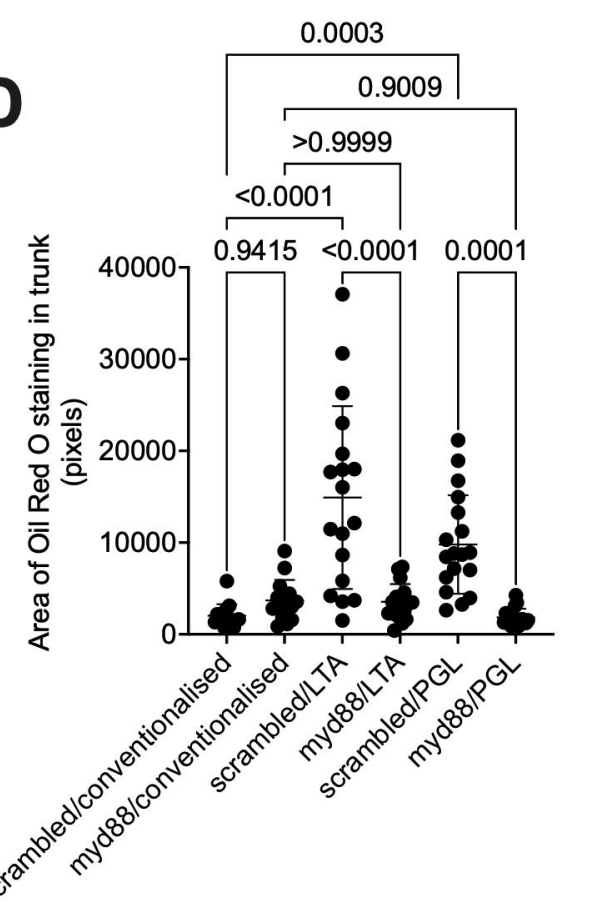

